\title{
A High-Order Dual-Time Stepping FR/CPR Method for Unsteady Incompressible Navier-Stokes Equations on Unstructured Moving Grids
}

\author{
Lai Wang ${ }^{1}$, Meilin $\mathrm{Yu}^{2}(\bowtie)$ \\ Department of Mechanical Engineering \\ University of Maryland, Baltimore County (UMBC), Baltimore, MD 21250
}

\begin{abstract}
A high-order accurate flux reconstruction/correction procedure via reconstruction (FR/CPR) method is developed to solve incompressible Navier-Stokes equations on unstructured moving grids. An artificial compressibility method is adopted to facilitate the common flux reconstruction on element interfaces. For unsteady flow simulations, a dualtime stepping method is used for temporal discretization. For simulations on deformable/moving grids, the Geometric Conservation Law (GCL) introduced in dynamic spatial coordinate transformation has been enforced. The newly developed method is verified with several steady and unsteady benchmark incompressible flow problems.
\end{abstract}

\section{Introduction}

Reliable and efficient simulation of unsteady incompressible flows is of great importance due to its wide applications to engineering problems such as biological engineering and wind/hydro energy harvesting. Since the flows in these problems are usually vortex-dominated and even turbulent, both robust and efficient spatial and temporal discretization methods with low numerical dissipation are needed to obtain reliable results at reasonable cost.

The primary difficulty of solving incompressible Navier-Stokes equations is due to the lack of time derivatives in the continuity equation which makes it impossible to directly march in time iteration. Historically, pressure-based methods, such as the SIMPLE-type ${ }^{[1]}$ and projection methods ${ }^{[2]}$, are extensively used to simulate the incompressible flows. These methods deal with the incompressible flow with pressure correction. Both of them solve the momentum equations first and then use a pressure correction to ensure a divergence free flow system. With these methods, the second-order accuracy can be achieved which is usually dissipative for study of turbulent flow or capturing details of flow structures when the mesh is coarse. Although many modern high-order numerical methods have been developed to handle vortex-dominated or turbulent flows, they are usually devised for the compressible flows. Thus, they can't be directly adopted for incompressible flow simulation using the traditional pressure based methods. When incompressible flows involve turbulence, especially when moving grid is used, different scales of both time and space structures need to be studied for better performance of engineering design. Therefore, it is desirable to take full advantage of the matured simulation techniques for compressible flows to facilitate the simulation of incompressible flows.

Artificial compressibility methods relax the constraint of incompressibility by adding a pseudo pressure variant with respect to time to the continuity equation which is firstly proposed by Chorin ${ }^{[3]}$. In this method, a new hyperbolic system is created which is similar to that governing the evolution of compressible flows. More applications based on Chorin's work can be found in $[4,5]$. Turkel ${ }^{[6]}$ has reviewed another method, namely, the preconditioning method, for compressible flows approaching the incompressible limit. To some extent, preconditioning methods ${ }^{[7]}$ also introduce artificial compressibility to incompressible flows. In this paper, for unsteady flows, a dual time stepping methods is used to march the flow system. For the outer time loop $t$, which is physical time loop, a second order backward differentiation formula (BDF2) method is employed. And in the inner time loop $\tau$, which is the pseudo-time loop, a pressure variant with respect to pseudo-time which achieves the artificial compressibility is added.

High-order methods like discontinuous Galerkin method $(\mathrm{DG})^{[8,9]}$, weighted essentially non-oscillatory scheme (WENO) ${ }^{[10]}$ and spectral difference method $(\mathrm{SD})^{[11]}$ have been studied in context of incompressible flows. Flux reconstruction/correction procedure via reconstruction $(\mathrm{FR} / \mathrm{CPR})^{[12-15]}$ is a recently developed method of high-order accuracy for unstructured grids. FR/CPR involves Lagrange interpolation as part of the flux reconstruction. Besides,

\footnotetext{
${ }^{1}$ Graduate Student, Department of Mechanical Engineering, email: bx58858@umbc.edu

${ }^{2}$ Assistant Professor, Department of Mechanical Engineering, AIAA Member, email: mlyu@umbc.edu
} 
it uses particular polynomials as correction functions to enforce the communication among adjacent elements and achieve higher accuracy than the original SD method ${ }^{[16]}$. Distribution of solution points does not cast any influence on the linear stability of the numerical method. As pointed out by Huynh ${ }^{[12,13]}$, with appropriate correction functions, DG can be recovered in the differential form as a specific instance of FR/CPR. FR/CPR does not involve any integral and is simpler than the traditional DG method while retains the high-order accuracy. The flexibility of unstructured grids for complicated geometry makes the numerical method capable of handling vast range of engineering applications.

In current study, an arbitrary Lagrangian-Eulerian (ALE) method is used to handle the moving/deformable grid. In order to enforce the Geometric Conservation Law (GCL), the work in Ref. [17] use the same discretization method as that applied to the original governing equation to solve the GCL equations every time step. This enforcement of GCL involves extra computational cost. But in present study, a source term considering GCL of grid motion is augmented to the governing equation in the computational domain ${ }^{[18]}$. The source term will cancel the geometry variant with time arising from deformable mesh.

The reminder of the paper is organized as follows. In Part 1 of Section II, the original governing equations of incompressible flows are introduced. After that, the artificial compressibility formulation is elaborated, and the new hyperbolic flow system using the artificial compressibility method is derived. The involvement of the moving grid into the governing equation is also presented here. A brief introduction of the FR/CPR spatial discretization method on quadrilateral elements is then shown in Part 2 of this section. A brief clarification on GCL is stated in Part 3 of this section. Finally, the dual-time stepping approach is introduced in Part 4 of Section II. In Section III, results of several benchmark tests are shown and discussed. Section IV concludes the paper.

\section{Governing Equations}

\section{Numerical Method}

The governing equations of unsteady incompressible flows can be expressed as

$$
\mathbf{I}_{i n c} \frac{\partial \mathbf{q}}{\partial t}+\frac{\partial\left(\mathbf{f}-\mathbf{f}_{v}\right)}{\partial x}+\frac{\partial\left(\mathbf{g}-\mathbf{g}_{v}\right)}{\partial y}=0,
$$

where $\mathbf{q}$ is the primitive variables and $\mathbf{f}, \mathbf{g}$ are the inviscid flux vectors and $\mathbf{f}_{v}, \mathbf{g}_{v}$ are the viscous flux vectors.

$$
\begin{gathered}
\mathbf{q}=\left(\begin{array}{l}
p \\
u \\
v
\end{array}\right), \mathbf{f}=\left(\begin{array}{c}
u \\
u^{2}+p / \rho \\
u v
\end{array}\right), \mathbf{g}=\left(\begin{array}{c}
v \\
u v \\
v^{2}+p / \rho
\end{array}\right), \\
\mathbf{f}_{v}=\left(\begin{array}{c}
0 \\
v(\partial u / \partial x) \\
v(\partial v / \partial x)
\end{array}\right), \mathbf{g}_{v}=\left(\begin{array}{c}
0 \\
v(\partial u / \partial y) \\
v(\partial v / \partial y)
\end{array}\right) .
\end{gathered}
$$

Herein, $\rho$ is density, $p$ is pressure, $u, v$ are velocities in $x, y$ direction respectively, $v$ is the kinetic viscosity. Note that

$$
\mathbf{I}_{\text {inc }}=\left(\begin{array}{ccc}
0 & 0 & 0 \\
0 & 1 & 0 \\
0 & 0 & 1
\end{array}\right),
$$

which makes it obvious that there is no time derivative in the continuity equation of (1). For artificial compressibility method, add the derivatives of primitive variables with respect to pseudo-time $\tau$. Equation (1) can be written as

$$
\mathbf{I}_{i n c} \frac{\partial \mathbf{q}}{\partial t}+\mathbf{I}_{a c} \frac{\partial \mathbf{q}}{\partial \tau}+\frac{\partial\left(\mathbf{f}-\mathbf{f}_{v}\right)}{\partial x}+\frac{\partial\left(\mathbf{g}-\mathbf{g}_{v}\right)}{\partial y}=0
$$

where

$$
\mathbf{I}_{a c}=\left(\begin{array}{ccc}
1 / \beta & 0 & 0 \\
0 & 1 & 0 \\
0 & 0 & 1
\end{array}\right),
$$

and $\beta$ is the artificial compressibility parameter. Usually, we rewrite (5) as 


$$
\mathbf{I}_{i n c} \frac{\partial \mathbf{q}}{\partial t}+\frac{\partial \mathbf{q}}{\partial \tau}+\frac{\partial\left(\mathbf{f}^{\prime}-\mathbf{f}_{v}\right)}{\partial x}+\frac{\partial\left(\mathbf{g}^{\prime}-\mathbf{g}_{v}\right)}{\partial y}=0
$$

where

$$
\mathbf{f}^{\prime}=\left(\begin{array}{c}
\beta u \\
u^{2}+p / \rho \\
u v
\end{array}\right), \mathbf{g}^{\prime}=\left(\begin{array}{c}
\beta v \\
u v \\
v^{2}+p / \rho
\end{array}\right) .
$$

For the following discussions, we will neglect the superscript of inviscid flux $\mathbf{f}^{\prime}, \mathbf{g}^{\prime}$. For a steady case, iterations can be done along the pseudo-time directly to achieve convergence without concerning the derivatives with respect to physical time. For unsteady cases, dual time stepping is straightforward for (7).

Transform equation (7) of physical domain $(t, x, y)$ into computational domain $(\tilde{t}, \xi, \eta)$ (Mesh only deforms in terms of physical time or computational time, and we can treat $\tilde{t}=t$.) Thus, we can have

$$
\mathbf{I}_{i n c} \frac{\partial \mathbf{Q}}{\partial t}+\frac{\partial \mathbf{Q}}{\partial \tau}+\frac{\partial \mathbf{F}}{\partial \xi}+\frac{\partial \mathbf{G}}{\partial \eta}=0
$$

in which

$$
\left\{\begin{array}{l}
\mathbf{Q}=|\mathbf{J}| \mathbf{q} \\
\mathbf{F}=|\mathbf{J}|\left(\mathbf{I}_{i n c} \mathbf{q} \xi_{t}+\mathbf{I}_{\rho} \mathbf{q} \xi_{t}+\left(\mathbf{f}-\mathbf{f}_{v}\right) \xi_{x}+\left(\mathbf{g}-\mathbf{g}_{v}\right) \xi_{y}\right), \\
\mathbf{G}=|\mathbf{J}|\left(\mathbf{I}_{i n c} \mathbf{q} \eta_{t}+\mathbf{I}_{\rho} \mathbf{q} \xi_{t}+\left(\mathbf{f}-\mathbf{f}_{v}\right) \eta_{x}+\left(\mathbf{g}-\mathbf{g}_{v}\right) \eta_{y}\right)
\end{array}\right.
$$

and $\mathbf{J}$ is the Jacobian matrix of coordinate transformation

$$
\mathbf{J}=\frac{\partial(x, y, t)}{\partial(\xi, \eta, \tilde{t})}=\left(\begin{array}{lll}
x_{\xi} & x_{\eta} & x_{t} \\
y_{\xi} & y_{\eta} & y_{t} \\
0 & 0 & 1
\end{array}\right) .
$$

$\mathbf{I}_{\rho}$ is defined by

$$
\mathbf{I}_{\rho} \mathbf{q}=\left(\begin{array}{ccc}
\tilde{\rho} & 0 & 0 \\
0 & 0 & 0 \\
0 & 0 & 0
\end{array}\right) .
$$

Since density of incompressible flow is a constant, $\tilde{\rho}$ can be any non-zero constant. But in order to couple the convergence of continuity equation with artificial compressibility parameter, in present study, $\tilde{\rho}=\beta$.

Note that the expression of the grid velocity is $\mathbf{v}_{g}=\left(x_{t}, y_{t}\right)$. Hence, we can have

$$
\left\{\begin{array}{l}
\xi_{t}=-\mathbf{v}_{g} \nabla \xi \\
\eta_{t}=-\mathbf{v}_{g} \nabla \eta
\end{array}\right.
$$

Then, the generalized Jacobian matrix $\mathbf{A}_{i}\left(\mathbf{A}_{i}=\partial \mathbf{F}_{\text {invs }} / \partial \mathbf{Q}, \partial \mathbf{G}_{\text {invs }} / \partial \mathbf{Q}\right.$ for $\left.i=1,2\right)$ of inviscid flux is

where $k^{i}=\xi, \eta$, for $i=1,2$ and

$$
\mathbf{A}_{i}=\left(\begin{array}{lll}
k_{t}^{i} & k_{x}^{i} \beta & k_{y}^{i} \beta \\
k_{x}^{i} / \rho & \Theta+k_{x}^{i} u+k_{t}^{i} & k_{y}^{i} u \\
k_{y}^{i} / \rho & k_{x}^{i} v & \Theta+k_{y}^{i} v+k_{t}^{i}
\end{array}\right),
$$

$$
\Theta=k_{x}^{i} u+k_{y}^{i} v .
$$

For incompressible flow, hyperbolic property of the inviscid flux no longer exits. But when artificial compressibility is introduced to incompressible flows, eigenvalues of Jacobi matrix $\mathbf{A}_{i}$ are

$$
\lambda_{j}\left(\mathbf{A}_{i}\right)=\Theta+k_{i}^{t} / 2, \Theta+k_{i}^{t} / 2+c, \Theta+k_{i}^{t} / 2-c \text { for } j=1,2,3,
$$

where $c$ is given by 


$$
c=\sqrt{\beta / \rho+\left(\Theta+k_{t}^{i} / 2\right)^{2}} .
$$

The hyperbolic property of inviscid flux is recovered and is very similar to the compressible flows. Thus, modern high-order numerical methods for compressible flows can be casted on incompressible flows with artificial compressibility.

\section{Spatial Discretization}

Refs. $[12,13]$ have shown that discontinuous Galerkin (DG) methods can be recovered by FR/CPR when Radau polynomials are selected as correction functions. FR/CPR-DG does not involve any integrals which makes it simpler and more efficient than traditional DG implementations. A brief review of FR/CPR is shown for completeness. Taking hexahedral element into account, transform the unstructured grid into a standard cell $[-1,1]^{2}$. With correction, the flux can be written as

$$
\left\{\begin{array}{l}
\hat{\mathbf{F}}=\mathbf{F}(\xi, \eta)+\left(\mathbf{F}_{c o m, L}-\mathbf{F}(-1, \eta)\right) g_{L}(\xi)+\left(\mathbf{F}_{c o m, R}-\mathbf{F}(1, \eta)\right) g_{R}(\xi) \\
\hat{\mathbf{G}}=\mathbf{G}(\xi, \eta)+\left(\mathbf{G}_{c o m, L}-\mathbf{G}(\xi,-1)\right) g_{L}(\eta)+\left(\mathbf{G}_{c o m, R}-\mathbf{G}(\xi, 1)\right) g_{R}(\eta)
\end{array}\right.
$$

where $\mathbf{F}, \mathbf{G}$ are the Lagrange interpolation in two directions,

$$
\left\{\begin{array}{l}
\mathbf{F}(\xi, \eta)=\sum_{i, j=1}^{N} \mathbf{F}_{i, j} \phi_{\xi, i} \phi_{\eta, j} \\
\mathbf{G}(\xi, \eta)=\sum_{i, j=1}^{N} \mathbf{G}_{i, j} \phi_{\xi, i} \phi_{\eta, j}
\end{array} .\right.
$$

Herein, $N$ is the number of solution points in each direction and $\phi$ is the base of Lagrange interpolation with $N$ points. $g_{L}, g_{R}$ are the correction functions. Similarly, $g_{\mathrm{L}}, g_{\mathrm{R}}$ have the same dimension-by-dimension form as

$$
g_{L / R}\left(\xi_{i}\right), i=1,2 \text {, with } \xi_{1}=\xi, \quad \xi_{2}=\eta \text {. }
$$

So we can simply regard the reconstructed flux of CPR method as two parts, the local flux and correction flux,

$$
\left\{\begin{array}{l}
\hat{\mathbf{F}}=\mathbf{F}^{L}+\mathbf{F}^{C} \\
\hat{\mathbf{G}}=\mathbf{G}^{L}+\mathbf{G}^{C}
\end{array} .\right.
$$

Common flux at the cell interface can be calculated (taking the Rusanov flux as an example) by

$$
\left\{\begin{array}{l}
\mathbf{F}_{\text {com }}=\frac{1}{2}\left(\mathbf{F}_{L}+\mathbf{F}_{R}\right)-\frac{1}{2}\left|\lambda^{\xi}\right|_{\max }\left(\mathbf{Q}_{R}-\mathbf{Q}_{L}\right)|J||\nabla \xi| \operatorname{sign}(\vec{n} \cdot \nabla \xi) \\
\mathbf{G}_{\text {com }}=\frac{1}{2}\left(\mathbf{G}_{L}+\mathbf{G}_{R}\right)-\frac{1}{2}\left|\lambda^{\eta}\right|_{\max }\left(\mathbf{Q}_{R}-\mathbf{Q}_{L}\right)|J||\nabla \eta| \operatorname{sign}(\vec{n} \cdot \nabla \eta)
\end{array},\right.
$$

where $\mathbf{F}_{L}=\mathbf{F}\left(\mathbf{Q}_{L}\right), \mathbf{F}_{R}=\mathbf{F}\left(\mathbf{Q}_{R}\right), \mathbf{Q}_{L}, \mathbf{Q}_{R}$ are the interpolation values of $\mathbf{Q}$ at the cell interfaces using (18). More details about the FR/CPR method can be found in Refs. [12-14].

On Substituting (18) into (9), we have

$$
\mathbf{I}_{i n c} \frac{\partial \mathbf{q}}{\partial t}+\frac{\partial \mathbf{q}}{\partial \tau}-\mathbf{I}_{i n c}\left(v_{g, x} \frac{\partial \mathbf{q}}{\partial x}+v_{g, y} \frac{\partial \mathbf{q}}{\partial y}\right)+\left(\frac{\partial \mathbf{f}^{L}}{\partial x}+\frac{\partial \mathbf{g}^{L}}{\partial y}\right)+\frac{1}{|\mathbf{J}|}\left(\frac{\partial \mathbf{F}^{C}}{\partial \xi}+\frac{\partial \mathbf{G}^{C}}{\partial \eta}\right)=0 .
$$

Herein, $v_{g, x}$ and $v_{g, y}$ are the velocity of grid deformation in $x$ and $y$ directions respectively. The fifth term of (23) is simply the correction flux using normal fluxes of cell surfaces.

\section{Geometric Conservation Law}

In Eq. (10),

$$
\frac{\partial|\mathbf{J}| \mathbf{q}}{\partial t}=|\mathbf{J}| \frac{\partial \mathbf{q}}{\partial t}+\mathbf{q}\left(\frac{\partial|\mathbf{J}|}{\partial t}+\frac{\partial|\mathbf{J}|}{\partial \xi} \xi_{t}+\frac{\partial|\mathbf{J}|}{\partial \eta} \eta_{t}\right) .
$$

In Ref. [17], the discretization is used to approximate the second term of right hand side of equation (24) to enforce the geometric conservation law. In present study, the above procedure involves the grid motion in the flux term $\mathbf{F}, \mathbf{G}$ while in equation (23), the grid motion exist in the correction term $\mathbf{F}^{C}$ and $\mathbf{G}^{C}$. Note that this part can be regard as a source term.

\section{Dual time stepping}

Eq. (23) can be rewritten as 


$$
\frac{\partial \mathbf{q}}{\partial \tau}+\mathbf{R}=0
$$

where $\mathbf{R}$ is the residual

$$
\mathbf{R}=-\left(\mathbf{I}_{i n c} \frac{\partial \mathbf{q}}{\partial t}-\mathbf{I}_{i n c}\left(v_{g, x} \frac{\partial \mathbf{q}}{\partial x}+v_{g, y} \frac{\partial \mathbf{q}}{\partial y}\right)+\left(\frac{\partial \mathbf{f}^{L}}{\partial x}+\frac{\partial \mathbf{g}^{L}}{\partial y}\right)+\frac{1}{|\mathbf{J}|}\left(\frac{\partial \mathbf{F}^{C}}{\partial \xi}+\frac{\partial \mathbf{G}^{C}}{\partial \eta}\right)\right) .
$$

$\mathrm{BDF} 2$ is used to approximate

$$
\frac{\partial \mathbf{q}}{\partial t}=\frac{3 \mathbf{q}^{n+1}-4 \mathbf{q}^{n}+\mathbf{q}^{n-1}}{2 \Delta t} .
$$

The first order backward Euler method is employed for the pseudo-time iterations.

More details on the development of the FR/CPR method for the incompressible flow on the moving mesh can be found in Ref. [19].

\section{Numerical Results}

In this section, several benchmark cases are presented to verify the developed incompressible flow solver. Steady cases, i.e. Kovasznay problem and Taylor-Couette problem, are simulated to test the performance of the solver for steady flow simulation. For the unsteady case, method of manufactured solution is used to test how the solver performs with moving mesh. In terms of practical application, flow past oscillation circular cylinder and airfoil are simulated. During the testing processes the fact that the numerical results are not sensitive with respect to the value of $\beta$ is noticed. But further study of this is not in the focus of the current paper.

\section{Kovasznay problem}

The analytical expressions for the pressure and velocity fields of the Kovasznay problem ${ }^{[9]}$ can be written as

$$
\left\{\begin{array}{l}
p(x, y)=-\frac{e^{2 \lambda x}}{2}, \\
u(x, y)=1-e^{\lambda x} \cos (2 \pi y), \\
v(x, y)=\frac{\lambda}{2 \pi} e^{\lambda x} \sin (2 \pi y),
\end{array}\right.
$$

with $\lambda=\operatorname{Re} / 2-\sqrt{\operatorname{Re}^{2} / 4+4 \pi^{2}}$, where $\operatorname{Re}$ is the Reynolds number of the flow. In current study, the Reynolds number is fixed at 5. The computational domain is $(-0.5,1.5) \times(0,2)$. Grid refinement study is carried out for $P^{2}, P^{3}$, and $P^{4}$ reconstruction. The convergence criterion is that the residual of the continuity equation converges more than 10 orders relative to the initial one. For this case, the value of $\beta$ is 1 .

The convergence rate of $v$ and $V_{\text {total }}$ are insistent with each other generally. But there are exceptions for this case. For example, when the grid is refined from to $16 \times 16$ and $P^{2}$ reconstruction is used, the $L_{2}$ error of $v$ decreases from $6.983 \times 10^{-3}$ to $7.815 \times 10^{-3}$ while that of total velocity decreases from $1.487 \times 10^{-2}$ to $1.140 \times 10^{-3}$. In terms of total velocity, convergence rate is 3.6 which is bigger than that of $\mathrm{v}$ which is 3.2 . Generally speaking, the solver performs as expected. Also, note that with a $32 \times 32$ mesh, $P^{4}$ reconstruction can make the error below $10^{-7}$. Numerical results verified the high-order accuracy property of the solver. 


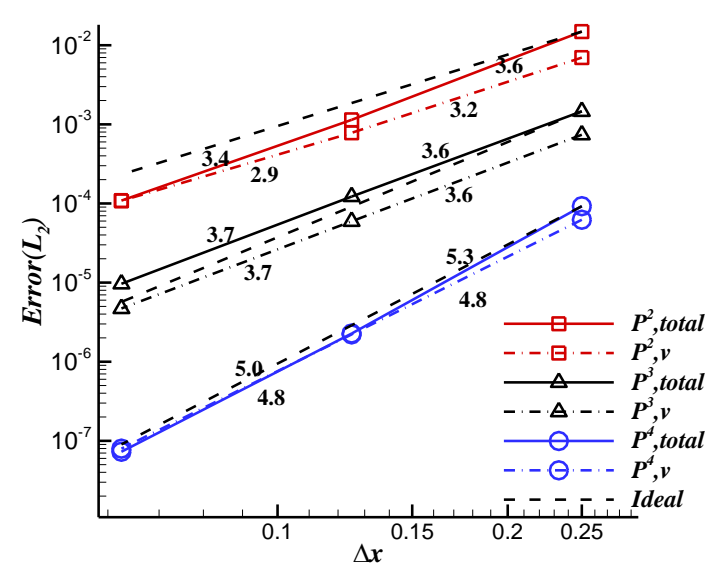

(a)

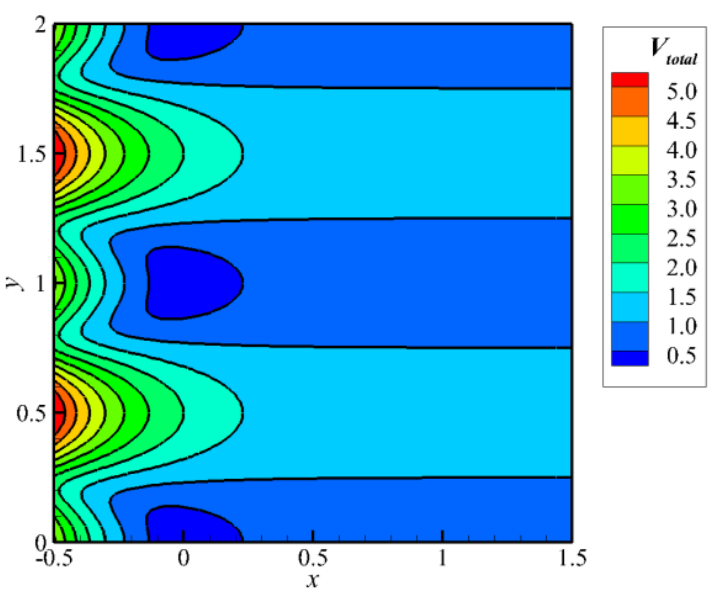

(b)

Figure 1 (a) Grid refinement study. Dash-dotted lines are the $L_{2}$ errors of velocity component $v$ in $y$ direction. Solid lines are the $L_{2}$ errors of total velocity. Square, triangle and circle symbols stand for $P^{2}, P^{3}$, and $P^{4}$ reconstruction respectively. Dash lines without symbols are the ideal slopes. (b) The velocity field $V_{\text {total }}=\sqrt{u^{2}+v^{2}}$ calculated using $P^{4}$ reconstruction on a $32 \times 32$ mesh.

\section{Taylor-Couette flow}

Taylor-Couette flow ${ }^{[11]}$ describes flow in the annulus between two cylinders which is induced by the different rotation speed of inner and outer cylinders. In our test, inner cylinder is rotating while the outer one is fixed. Given the rotation speed of inner cylinder is $\omega$ and the radius of inner and outer cylinders are $r_{1}, r_{2}$ respectively. The analytical solution of the velocity field can be expressed as

$$
\left\{\begin{array}{l}
u(x, y)=V_{\theta} \sin \theta \\
v(x, y)=-V_{\theta} \cos \theta
\end{array}\right.
$$

Herein, $\theta$ is the angle to $\mathrm{x}$ axis and

$$
V_{\theta}=r_{1} \omega_{1}\left(\frac{r_{2}}{r}-\frac{r}{r_{2}}\right) /\left(\frac{r_{2}}{r_{1}}-\frac{r_{1}}{r_{2}}\right)+r_{2} \omega_{2}\left(\frac{r}{r_{1}}-\frac{r_{1}}{r}\right) /\left(\frac{r_{2}}{r_{1}}-\frac{r_{1}}{r_{2}}\right)
$$

In this case, $\omega_{1}=1, \omega_{2}=0$. Cells at the inner and outer circular surfaces involve curved edges. In this case, $P^{4}$ elements are used. And artificial compressibility parameter $\beta=10$. Also grid refinement study are carried out for $P^{2 \sim 5}$ reconstructions. Since for this case the error of velocity components in $x, y$ directions share sort of symmetrical similarity, figure 2(a) shows the errors of $u$ only. Figure 2(b) is the total velocity field on mesh $20 \times 3,20$ cells in circumferential direction and 3 cells in radial direction. The results can be a proof of the solver's capability of handling curved boundaries.

\section{Method of manufactured solution with moving mesh}

To make the solutions of incompressible Navier-Stokes equations are

$$
\left\{\begin{array}{l}
p(x, y)=\sin \left(f_{s} \pi x-f_{s} \pi y+f_{t} \pi t\right), \\
u(x, y)=\sin \left(f_{s} \pi x+f_{t} \pi t\right) \sin \left(f_{s} \pi y+f_{t} \pi t\right), \\
v(x, y)=\cos \left(f_{s} \pi x+f_{t} \pi t\right) \cos \left(f_{s} \pi y+f_{t} \pi t\right),
\end{array}\right.
$$

Force terms(source terms) can be added to the original governing equations, since it's really straight forward to get the source terms. It won't be repeated here. In this test, $f_{s}=6, f_{t}=20$ and the computational domain is $[0,0.5] \times$ $[0,0.5]$.

For this test, the physical domain remains the same while the inner grids deform with time in following manner

$$
\left\{\begin{array}{l}
d x(t)=A_{x} L_{x} d t / t_{\max } \sin \left(n_{t} t\right) \sin \left(n_{x} \pi x\right) \sin \left(n_{y} \pi y\right) \\
d y(t)=A_{y} L_{y} d t / t_{\max } \sin \left(n_{t} t\right) \sin \left(n_{x} \pi x\right) \sin \left(n_{y} \pi y\right)
\end{array},\right.
$$

where $n_{t}=4 f_{t}, n_{x}=n_{y}=4, A_{x}=A_{y}=0.2, L_{x}=L_{y}=0.5, t_{\max }=0.05$ and $d t=1 \times 10^{-7}$. 
Simulations stop at $t=0.0125$, and in this process mesh deforms from figure to figure. $3^{\text {rd }}, 4^{\text {th }}, 5^{\text {th }} C P R-D G$ schemes are tested for this case. For this case, especially when $4^{\text {th }}$ and $5^{\text {th }}$ order schemes are used, a very small step size $\Delta t$ need to be used to make sure the error is spatial-discretization dominated, we basically run tests on relative coarser meshes for higher order of accuracy schemes on behalf of efficiency. Figure 4 shows the convergence rates with grid refinement, both stationary mesh and moving mesh are involved. It is easy to find out that our solver show an obvious tendency of approaching the optimal convergence rate when the grid becomes finer and finer.

Figure 5 shows the contours at the end moment of primitive variables. With deformable mesh, the solver still shows a good accuracy preserving property. Meanwhile, flow structure profiles are well preserved.

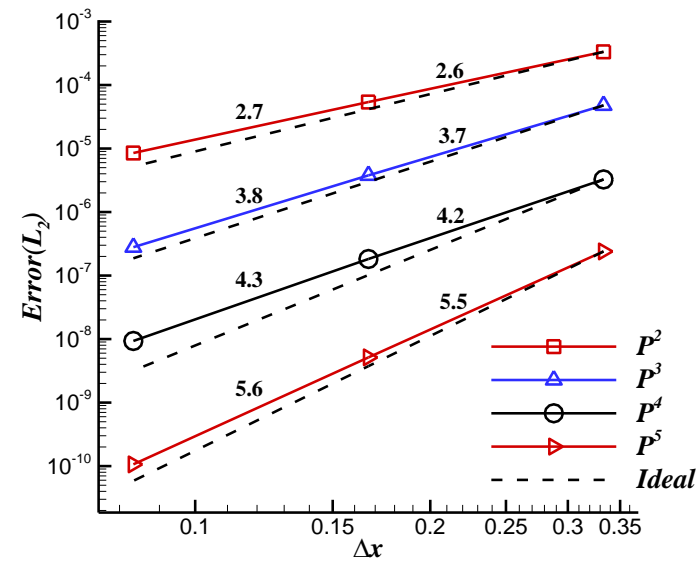

(a)

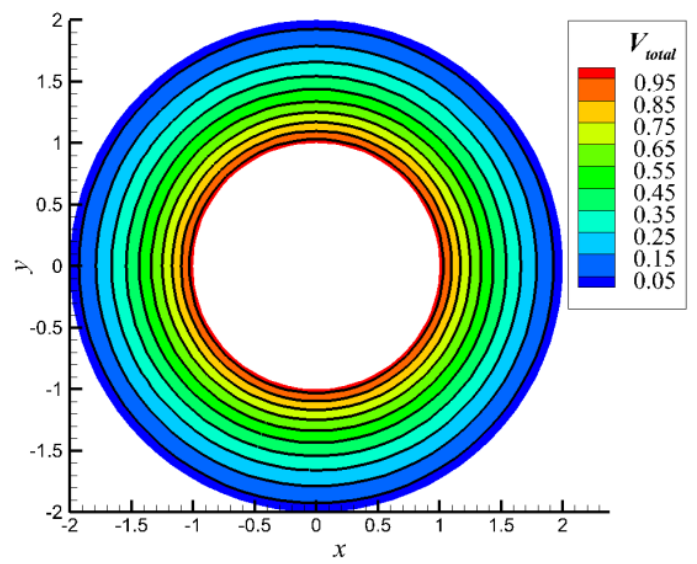

(b)

Figure 2 (a) Grid refinement study on errors of $u$; (b) Velocity field of Taylor-Couette flow on mesh $20 \times 3, P^{4}$ reconstruction

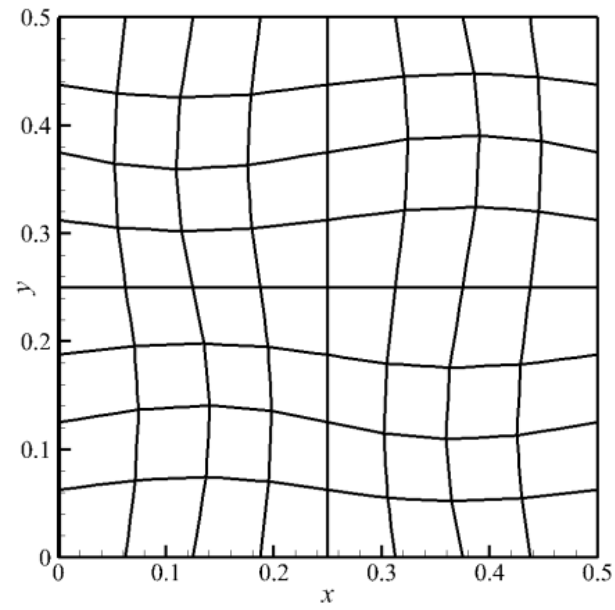

Figure 3 Mesh status at stop moment

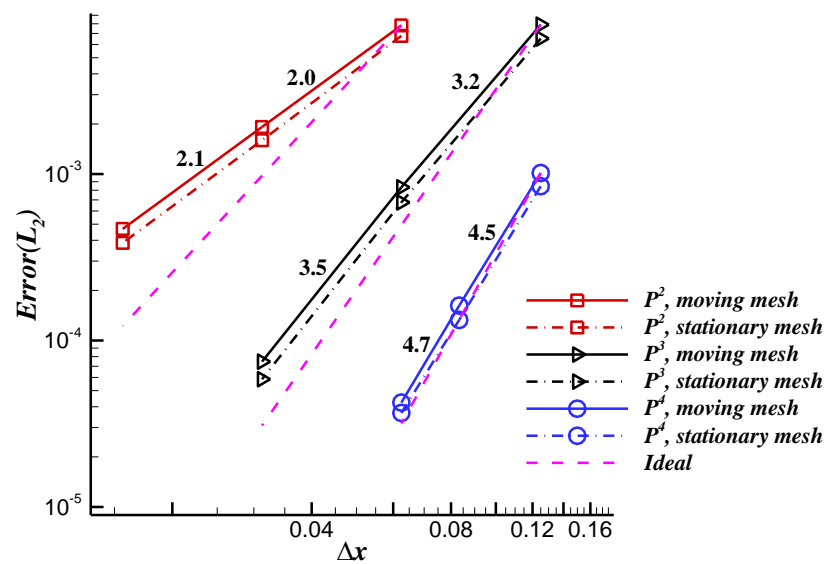

Figure 4 Grid refinement study on errors of total velocity 


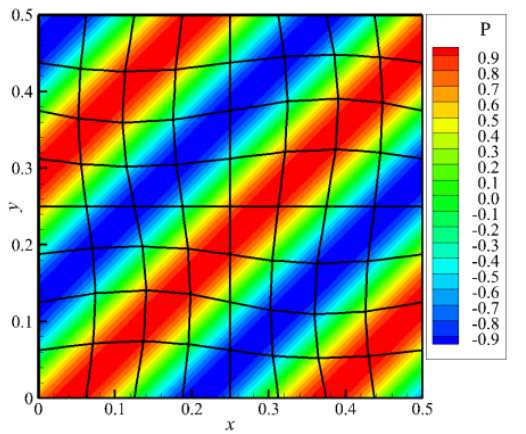

(a)

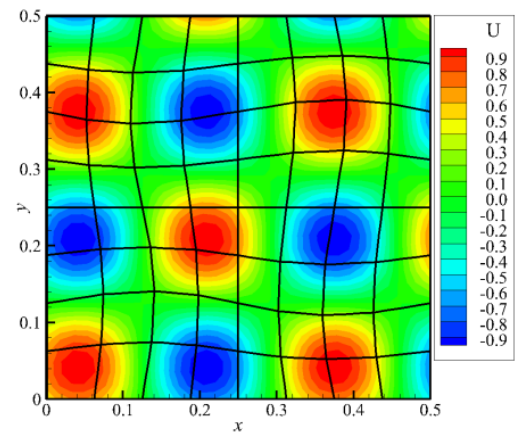

(b)

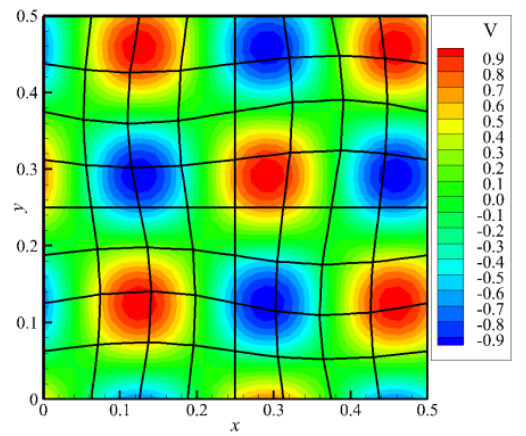

(c)

Figure 5 Contours of primitive variables on $8 \times 8$ mesh, $P^{4}$ reconstruction: (a) $p$;(b) $u$;(c) $v$

\section{Oscillating Cylinder}

Flow past a circular cylinder is a benchmark to test the capability of complex temporal and spatial flow structures. Flow past a fixed cylinder at Reynolds Number $(\operatorname{Re}) 100^{[20]}$ and $185^{[21]}$ are firstly tested to validate the code. Ref. [18] declares that simulations on deforming mesh with blending function and rigid motion of whole mesh domain shows much similar results. So in this paper, rigid motion of grid deformation is used. For $\mathrm{Re}=100$ case, a polynomial refinement is carried out on a relative coarse mesh as illustrated in figure $6,20 \times 24,20$ elements in normal direction, 6 elements in each side. The Strouhal number of this case matches well with previous works. But the lift and drag coefficients have some differences. This inconsistency, small as it is, may due to the value of $\beta$. In this case $\beta=1$, paper points out that for a relative small $\operatorname{Re}$, a larger $\beta$ is preferred. As mentioned previously, in present work, we do not discuss the effect of $\beta$. Figure 7 shows the contour of vorticity with a $P^{7}$ reconstruction, from which we can see the vortex shedding is captured fairly enough.

For the case with $\mathrm{Re}=185$, a grid refinement study is firstly carried out using the $P^{3}$ reconstruction. From table 2 , it is clear that Strouhal numbers from the current study agree well with those from previous studies. $C_{l}$ also matches former works while there is a difference for $C_{d}$. Figure 8(a) is the force coefficients history on $40 \times 96$ mesh when no oscillation is involved.

For the oscillating cylinder case, Re is set as 185 . Simulations are carried out on the $40 \times 96$ mesh. The cylinder motion is defined as $y=A_{e} \sin \left(2 \pi f_{e} t\right)$, where $A_{e}$ is the amplitude of the oscillation and $f_{e}$ is the oscillating frequency. Frequency ratio $f_{e} / f_{0}=0.8,1.2$ are tested, where $f_{0}$ is the frequency of natural vortex shedding. For this test, $f_{0}=0.192$ is chosen. Ref. [21] reported that when the ratio $f_{e} / f_{0}$ is smaller than one, the profile of both drag and lift history will preserve the periodic pattern and the vortex shedding frequency remains consistent with the cylinder oscillating frequency. Besides, when the ratio increases in this region, the amplitudes of lift and drag will increase. For ratio larger than 1, the profile of both lift and drag histories will have fluctuations and form wave packages. Figures $8(b, c)$ show an agreement with Ref. [21]'s work.

Table 1 Polynomial refinement of flow past a fixed cylinder, $\mathrm{Re}=100$

\begin{tabular}{|l|c|c|c|}
\hline \multicolumn{4}{|c|}{$\mathrm{Re}=100$} \\
\hline \multicolumn{1}{|c|}{ Method } & $S t$ & $C_{d}$ & $C_{l, \max }$ \\
\hline$P^{2}$ reconstruction & 0.1629 & 1.2581 & 0.3068 \\
\hline$P^{3}$ reconstruction & 0.1642 & 1.3195 & 0.3193 \\
\hline$P^{4}$ reconstruction & 0.1642 & 1.3205 & 0.3191 \\
\hline$P^{5}$ reconstruction & 0.1639 & 1.3185 & 0.3185 \\
\hline$P^{6}$ reconstruction & 0.1637 & 1.3189 & 0.3187 \\
\hline$P^{7}$ reconstruction $^{[20]}$ & 0.1637 & 1.3196 & 0.3189 \\
\hline Experiment & 0.163 & & 0.325 \\
\hline A. S. Chan, P. A. Deway, etc. ${ }^{[20]}$ & 0.164 & 1.338 & \\
\hline
\end{tabular}




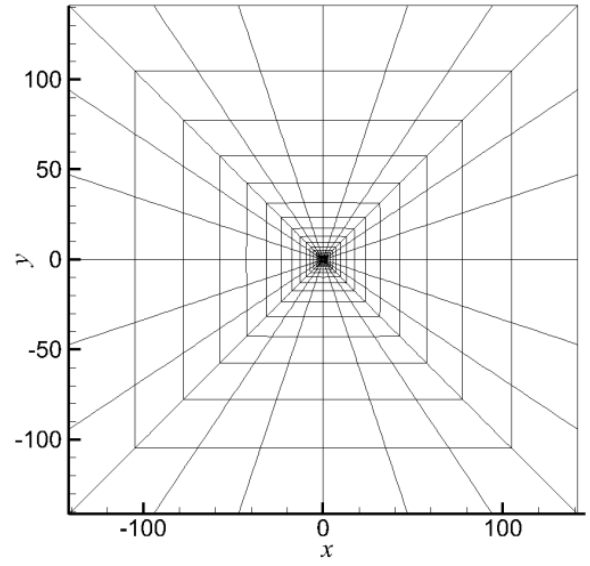

Figure 6 Mesh, 20×24, 20 elements in normal direction, 6 elements in each side

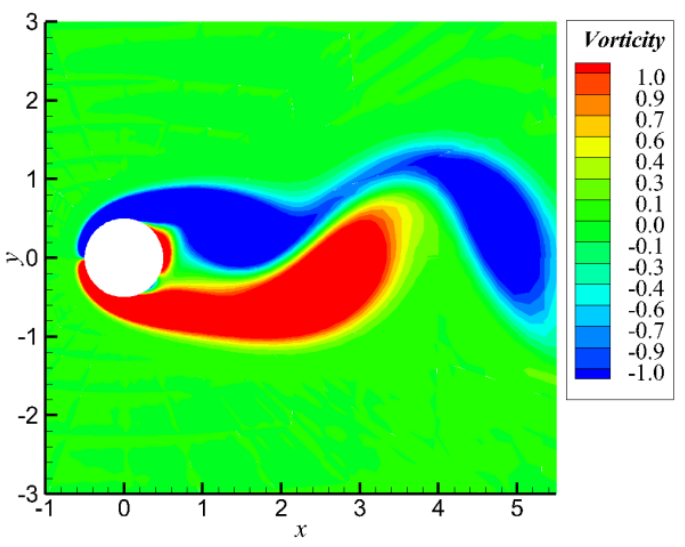

Figure 7 Vorticity contours of flow past a fixed cylinder, $\mathrm{Re}=100, P^{7}$ reconstruction

Table 2 Grid refinement of flow past a fixed cylinder, $\operatorname{Re}=185, P^{3}$ reconstruction

\begin{tabular}{|c|c|c|c|}
\hline Mesh & $S t$ & $C_{d}$ & $C_{l}$ \\
\hline $20 \mathrm{~s} 24$ & 0.1931 & 1.1762 & 0.3767 \\
\hline 30348 & 0.1916 & 1.3105 & 0.4402 \\
\hline 40496 & 0.1916 & 1.3159 & 0.4416 \\
\hline Experiment $^{[2]}$ & 0.19 & 1.28 & 0.443 \\
\hline E. Guilmineau, P. Queutey $^{[2]]}$ & 0.195 & 1.287 & 0.442 \\
\hline X. Y. Lu, C. Dalton & 1.31 & \\
\hline
\end{tabular}

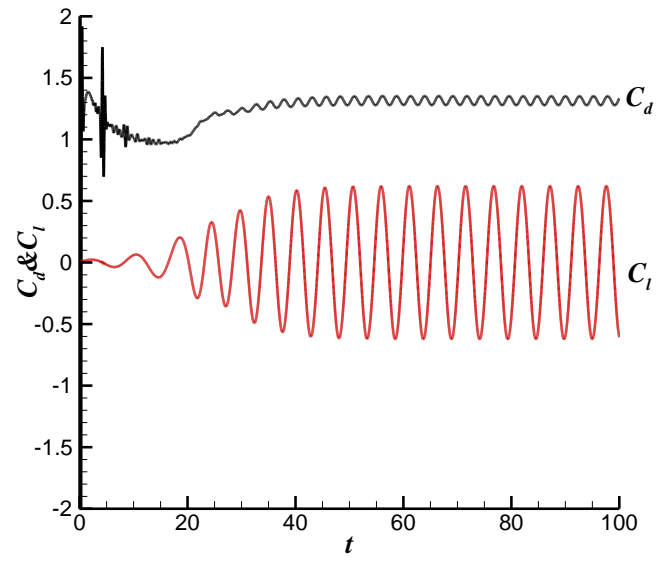

(a)

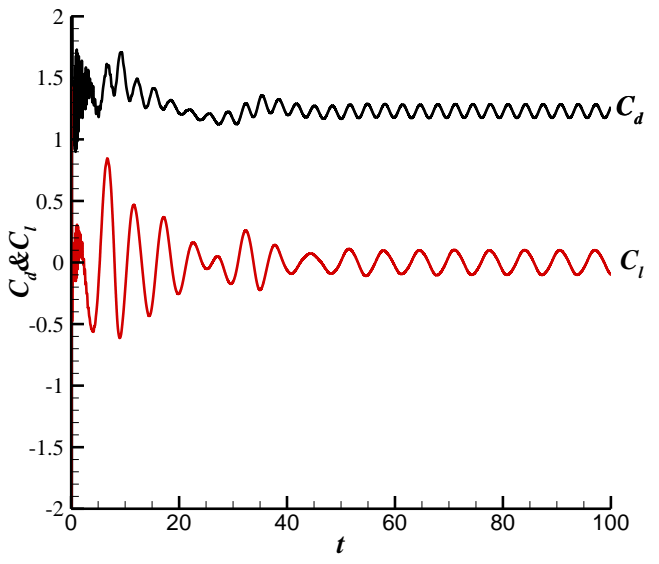

(b) 


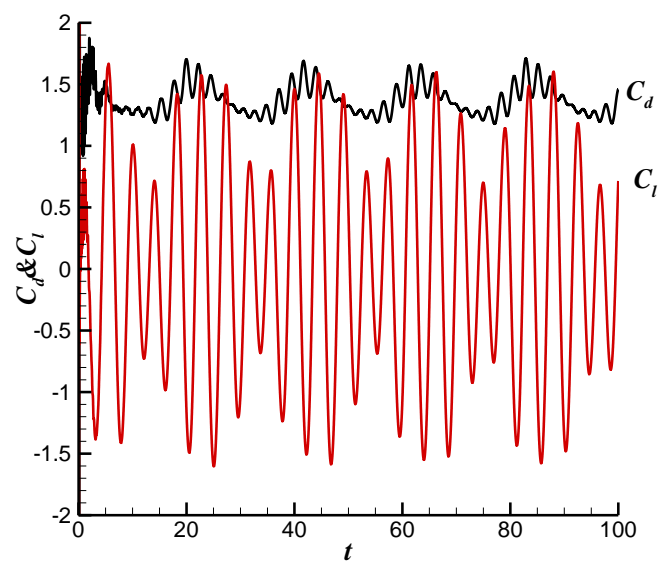

(c)

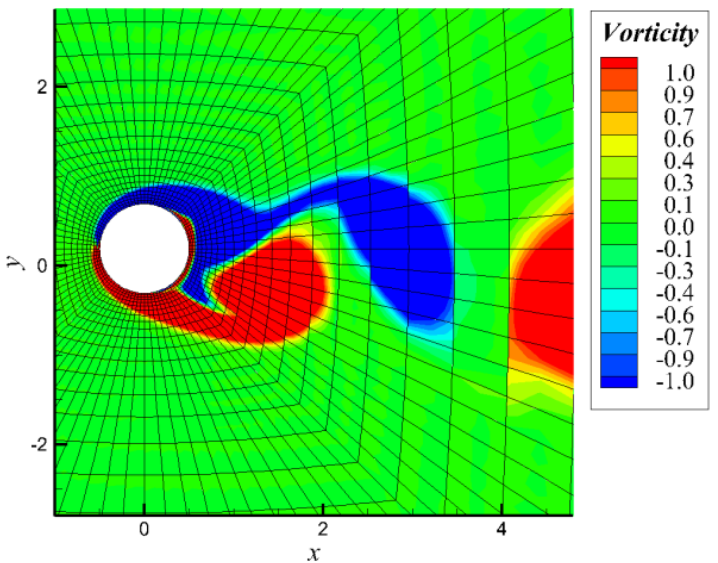

(d)

Figure $8 \mathrm{Re}=185$, mesh $40 \times 96, P^{3}$ reconstruction: (a). $C_{l}, C_{d}$ history of flow past a fixed cylinder, $\operatorname{Re}=185$; (b). $C_{l}, C_{d}$ history past an oscillating cylinder, $f_{e} / f_{0}=0.8$; (c). $C_{l}, C_{d}$ history past on oscillating cylinder, $f_{e} / f_{0}=1.2$; (d) Vorticity contour of $f_{e} / f_{0}=1.2$, cylinder is at the extreme upper position.

\section{Conclusions}

A high-order accurate flux reconstruction/correction procedure via reconstruction (FR/CPR) method is developed to solve incompressible Navier-Stokes equations on unstructured moving grids. An artificial compressibility method is adopted to facilitate the common flux reconstruction on element interfaces. For unsteady flow simulations, a dual-time stepping method is used for temporal discretization. From numerical tests, the newly developed solver works well for both steady cases and unsteady cases with grid motion.

\section{References}

[1] S. V. Patankar, D. B. Spalding. A Calculation Procedure for Heat, Mass and Momentum Transfer in Three-Dimensional Parabolic Flow. International Journal of Heat and Mass Transfer, vol. 15, issue 10, pp. 1787-1806, 1972.

[2] A. S. Almgren, J. B. Bell, P. Colella, L. H. Howell, M. L. Welcome. A conservative Adaptive Projection Method for The Variable Density Incompressible Navier-Stokes Equations. Journal of Computational Physics, vol. 142, issue 1, pp. 1-46, 1998.

[3] A. J. Chorin. A Numerical Method for Solving Incompressible Viscous Flow Problems. Journal of Computational Physics, vol. 2, issue 1, 12-26.

[4] S. E. Rogers. An Upwind-Differencing Scheme for the Incompressible Navier-Stokes Equations. Applied Numerical Mathematics, vol. 8, issue 1, 43-64, 1991.

[5] A. G. Malan, R. W. Lewis, P. Nithiarasu. An Improved Unsteady, Unstructured, Artificial Compressibility Finite Volume Scheme for Viscous Incompressible Flows: Part I .Theory and Implementation. International Journal for Numerical Methods in Engineering, vol. 54, issue 5, 695-714.

[6] E. Turkel. Preconditioned methods for Solving the Incompressible and Low Speed Compressible Equations. Journal of Computational Physics, vol. 72, issue 2, pp. 277-298, 1987.

[7] J. M. Weiss, W. A. Smith. Preconditioning Applied to Variable and Constant Density Flows. AIAA Journal, vol. 33, no. 11, pp. 2050-2057, 1995.

[8] A. Crivellini, V. D’Alessandro, F Bassi. Assessment of A High-Order Discontinuous Galerkin Method for Incompressible Three-Dimensonal Navier-Stokes Equations: Benchmark Results for The Flow Past A Sphere Up to $R e=500$. Computers \& Fluids, vol. 86, pp. 442-458, 2013.

[9] F. Bassi, A. Crivellini, D.A. Di Pietro, S. Rebay. An artificial Compressibility Flux for The Discontinuous Galerkin Solution of The Incompressible Navier-Stokes Equations. Journal of Computational Physics, vol. 218, issue 2, pp. 794-815, 2006.

[10] J. Y. Yang, S. C. Yang, Y. N. Chen, C. A. Hsu. Implicit Weighted ENO Schemes for the ThreeDimensional incompressible Navier-Stokes Equations. Journal of Computational Physics, vol. 146, 
issue 1, pp. 464-487, 1998.

[11] C. L. Liang, A. Chan, X. Liu, A. Jameson. An Artificial Compressibility Method for The Spectral Difference Solution of Unsteady Incompressible Navier-stokes Equations on Multiple Grids. AIAA Paper, AIAA-2011-48, 2011.

[12] H. T. Huynh. A Flux Reconstruction Approach to High-Order Schemes including Discontinuous Galerkin Methods. AIAA Paper, 4079, 2007.

[13] H. T. Huynh. High-Order Methods Including Discontinuous Galerkin by Reconstructions On Triangular Meshes. AIAA Paper, 44, 2011.

[14] Z. J. Wang, and H. Y. Gao. A Unifying Lifting Collocation Penalty Formulation Including the Discontinuous Galerkin, Spectral Volume/Difference Methods for Conservation Laws on Mixed Grids. Journal of Computational Physics, vol. 228, issue 21, pp. 8161-8186, 2009.

[15] P. E. Vincent, P. Castonguay and A. Jameson, A new class of high-order energy stable flux reconstruction schemes, Journal of Scientific Computing, vol. 47, no. 1, pp. 50-72, 2011.

[16] M. L. Yu, Z. J. Wang and Y. Liu. On the Accuracy and Efficiency of Discontinuous Galerkin, Spectral Difference and Correction Procedure via Reconstruction Methods. Journal of Computational Physics, vol. 259, 0;/pp. 70-95, 2014.

[17] K. Ou. A. Jameson. On the Temporal and Spatial Accuracy of Spectral Difference Method on Moving Deformable Grids and the Effect of Geometry Conservation Law. AIAA Paper, 5032, 2010.

[18] M. L. Yu, Z.J. Wang, H. Hu. A High-Order Spectral Difference Method for Unstructured Dynamic Grids. Computers \& Fluids, vol. 48, issue 1, pp. 84-97, 2011.

[19] M. L. Yu and L. Wang. A high-order flux reconstruction/correction procedure via reconstruction formulation for unsteady incompressible flow on unstructured moving grids, to be sumitted.

[20] A. S. Chan, P. A. Dewey, A. Jameson,et al. Vortex Supression and Drag Reduction in the Wake of Conter-rotating cylinders. Journal of Fluid Mechanics, vol. 679, pp. 343-382, 2011.

[21] E. Guilmineau, P. Queutey. A Numerical Simulation of Vortex Shedding from an Oscillating Cylinder. Journal of Fluids and Structures, vol. 16, issue 6, pp. 773-794, 2002.

[22] X. Y. Lu, C. Dalton. Calculation of the timing of vortex formation from an oscillating cylinder. Journal of Fluids and Structures, vol. 5, issue. 10, pp. 527-541, 1996. 